\title{
Matérista
}

Revista Matéria, v. 16, n. 4, pp. 819 - 829, 2011

ISSN 1517-7076

http://www.materia.coppe.ufrj.br/sarra/artigos/artigo11453

\section{Previsão de vida em tubos de caldeiras com base na medição da espessura da camada de óxido por ultrassom}

\author{
CARDOSO, B. R. , COMELI, F.W. II, SANTANA, R. M. I, FURTADO, H. C. ${ }^{\text {I }}$ \\ ${ }^{\text {I }}$ Centro de Pesquisas de Energia Elétrica - Cepel, CEP - 21941-911, Rio de Janeiro, RJ. \\ e-mail: brunorc@cepel.br ; heloisa@cepel.br ; roberta@cepel.br \\ II Tractebel Energia - CEP - 88745-000, Capivari de Baixo, SC. \\ e-mail: francowc@tractebelenergia.com.br
}

\begin{abstract}
RESUMO
Falhas em tubos de caldeiras são as maiores causas de paradas forçadas de unidades de geração termelétrica, sendo que a espessura da camada de óxido, que se forma na parede interna destes tubos é a principal causadora de falhas por sobreaquecimento. A partir dos valores medidos da camada interna de óxidos é possível estimar a vida residual do componente através de correlações com parâmetros já estabelecidos na comunidade científica. O Cepel vem desenvolvendo metodologias para estimativa de vida residual de componentes de caldeiras de usinas termelétricas, seguindo as tendências mundiais e as necessidades de seus clientes no Brasil. Com a recente aquisição de equipamento para detecção de defeitos e medições de espessura via ultrassom, tornou-se possível realizar medidas de espessura tanto da parede das tubulações das caldeiras como da camada interna de óxidos presente nas mesmas. O presente artigo apresenta uma comparação entre os resultados de medições de espessura de camada de óxidos em tubos pela técnica não destrutiva de ultrassom e pela técnica destrutiva de metalografia associada à microscopia ótica, em diferentes faixas de espessura da camada e análise do efeito do acabamento superficial na confiabilidade destes resultados por ultrassom. Finalmente, o trabalho analisa de maneira crítica os resultados de vida remanescente, obtidos a partir da medição espessura de camada de óxido. Para esta análise tomou-se como base tubos de uma mesma unidade geradora com diferentes valores de camada de óxido e com o mesmo tempo de operação.
\end{abstract}

Palavras chaves: Técnica ultrassônica, Camada de óxido, Vida remanescente.

\section{Expected life in tubes of boilers based on measurement of the thickness the oxide layer by ultrasonic}

\section{ABSTRACT}

Boiler tube failures are the major causes of forced shut downs in thermoelectric units. The thickness of the oxide layer formed on the inner wall of these tubes is the main cause of failure due to overheating. From the measured values of the inner oxide layer, it is possible to estimate the residual life of the component via correlations with parameters established in the scientific community. Cepel has been developing methodologies for estimating the residual life of boiler components for power plants, following the world trends and the needs of its customers in Brazil. With the recent acquisition of ultrasonic equipment for detecting defects and to perform thickness measurements it has become possible to perform both operations at the same time. This article presents a comparison between the results of measurements of oxide layer thickness in tubes by the non-destructive ultrasonic technique and the destructive metallographic technique associated with optical microscopy. Oxide layers with different thicknesses were used to perform the analysis. Also, the effect of surface preparation was evaluated for the reliability of results by ultrasonic testing. Finally, the study examines, in a critical way, the remaining life results obtained from the measured oxide layer thickness. To perform this analysis, two tubes with different oxide scale thicknesses were taken from a generation unit, both of them operated for the same amount of time.

Keywords: Ultrasonic technique, Oxide scale thickness, Remaining life. 


\section{INTRODUÇÃO}

A extensão de vida útil de componentes de caldeiras de plantas industriais é assunto de grande interesse e está diretamente ligada aos componentes que operam a altas temperaturas. Dentre os mecanismos de falha atuantes em tubulações e componentes de caldeiras que operam sob regime de fluência, o sobreaquecimento devido à formação da camada interna de óxido possui uma grande influência na vida dos componentes. A presença desses depósitos e filmes na parede dos tubos aumenta a temperatura do metal, o que acelera os mecanismos de dano, reduzindo drasticamente a vida do material [1].

A camada de óxido que se forma na superfície interna de tubos de aço baixa liga (até 3\% $\mathrm{Cr}$ ) expostos ao vapor gerado nas caldeiras consiste de uma camada com diferentes níveis. Quando a temperatura do metal está abaixo de aproximadamente $560^{\circ} \mathrm{C}$ e existe uma alta pressão parcial de oxigênio, uma camada dupla consistindo de um filme de Magnetita $\left(\mathrm{Fe}_{3} \mathrm{O}_{4}\right)$ e outro de Hematita $\left(\mathrm{Fe}_{2} \mathrm{O}_{3}\right)$ é encontrada. A altas temperaturas, uma camada adicional de Wustita $(\mathrm{FeO})$ pode aparecer. Se o aço do tubo possuir elementos de liga, um óxido spinel (Fe,Cr,Mo $)_{3} \mathrm{O}_{4}$, pode se formar, conforme a camada de óxidos cresce na direção da parede do tubo. Esses óxidos se formam de acordo com as seguintes reações [2]:

$$
\begin{aligned}
& \mathrm{Fe}(\mathrm{s})+1 / 2 \mathrm{O}_{2}(\mathrm{~g})=\mathrm{FeO}(\mathrm{s}) \\
& 3 \mathrm{FeO}(\mathrm{s})+1 / 2 \mathrm{O}_{2}(\mathrm{~g})=\mathrm{Fe}_{3} \mathrm{O}_{4}(\mathrm{~s}) \\
& 2 \mathrm{Fe}_{3} \mathrm{O}_{4}(\mathrm{~s})+1 / 2 \mathrm{O}_{2}(\mathrm{~g})=3 \mathrm{Fe}_{2} \mathrm{O}_{3}(\mathrm{~s})
\end{aligned}
$$

A pressão parcial de oxigênio necessária para que cada uma das reações expostas acima ocorra na faixa de temperaturas de 430 a $705^{\circ} \mathrm{C}$ pode ser estimada através de princípios termodinâmicos. Cálculos da literatura mostram que a Hematita requer pressões de oxigênio com dez ordens de grandeza maiores para se formar do que a Magnetita e a Wustita. Para que a Wustita mantenha-se estável, a pressão do oxigênio deve estar abaixo de um valor crítico (dependente da temperatura) e a temperatura deve estar acima de $560^{\circ} \mathrm{C}$. $\mathrm{Na}$ prática, a Magnetita é o óxido mais presente na superfície interna dos tubos em caldeiras de ciclo químico convencional [1].

Devido a flutuações na carga e o crescimento da camada interna de óxido durante a operação, é improvável que a temperatura do metal mantenha-se constante durante o serviço. É estabelecido como prática na indústria, estimar uma temperatura de metal média ou equivalente de operação através de métodos de observação de parâmetros tais como dureza, microestrutura e espessura de camada interna de óxido, sendo a última mais especificamente usada na avaliação de tubos. Como as mudanças nesses parâmetros são funções do tempo e da temperatura, os valores obtidos nas análises podem ser usados para estimar um histórico térmico para certo tempo de operação. A temperatura estimada pode então ser usada em conjunto com parâmetros de tensão de ruptura para estimar a vida remanescente.

No caso específico da camada interna de óxido de tubos de superaquecedores e reaquecedores de caldeiras, já existe um método consagrado na literatura [3].

O presente artigo apresenta uma metodologia para validação de medições de camada interna de óxido realizadas pela técnica ultrassônica. Além disso, o trabalho analisa de maneira crítica os resultados de vida remanescente, obtidos a partir da medição espessura de camada de óxido. Para esta análise tomou-se como base tubos de uma mesma unidade geradora com diferentes valores de camada de óxido e com o mesmo tempo de operação.

\section{MATERIAIS E MÉTODOS}

\subsection{Validação das Medições Realizadas com Equipamento Ultrassônico}

Até o início dos anos 80, a única forma de se medir a camada interna de óxido em tubos de superaquecedores e reaquecedores de caldeiras era através de ensaios destrutivos com a remoção de amostras. A camada de óxido era então medida em laboratório por meios de preparação metalográfica. Tomando-se os cuidados para evitar a quebra da camada durante a preparação da amostra, era possível obter uma medida precisa. Esta metodologia apresenta inúmeras desvantagens, pois a retirada e colocação de amostras consomem tempo e representam um custo à manutenção. 
A técnica ultrassônica mostra-se como uma boa alternativa ao método destrutivo de retirada de amostras. As medições podem ser realizadas em equipamentos que apresentem o modo de observação AScan, mais tradicional, que consiste na visualização dos ecos de reflexão do som nas interfaces da peça inspecionada.

A espessura da camada de óxido é calculada medindo-se o intervalo de tempo da onda ultrassônica entre o eco refletido na interface aço/camada de óxido e eco refletido na superfície interna do tubo. O eco da interface aço/camada de óxido é muito menor do que o eco da interface camada de óxido/ar ou líquido na superfície interna do tubo. A maior dificuldade da medição é separar estes ecos e medir o intervalo de tempo entre eles.

É importante ressaltar que a possibilidade de executar medições em tempo real diretamente no campo representa um ganho tecnológico que permite uma análise mais rápida dos resultados obtidos. HAWKINS et al. [4] descrevem uma metodologia adotada no final da década de 80 para se obter medições de camadas de óxido através da técnica ultrassônica e deixaram claro que esta era uma tarefa complexa e que dependia muito do avanço tecnológico. Foram descritas dificuldades em termos de se obter um transdutor de alta frequência que pudesse ser aplicado nas condições de campo, capaz de distinguir os dois ecos necessários para realizar a medição. Isso tornava necessário um tratamento de sinais que deveria ser realizado após a captura dos sinais ultrassônicos em um computador com o auxílio de um osciloscópio. Um sistema deste tipo mostrava-se preciso, no entanto não era conveniente para aplicações no campo, onde medições em tempo real são necessárias.

Outros trabalhos na área [5] comentam que a utilização de equipamentos ultrassônicos utilizados para medição de espessura de paredes de tubos não eram precisos o suficiente para realizar a tarefa, pois à medida que a que camada ficava mais espessa, a precisão diminuía. Desde então, diversos fabricantes vêm desenvolvendo equipamentos ultrassônicos capazes de medir a camada interna de óxido.

Diante da possibilidade de expandir a gama de ensaios não destrutivos que poderão auxiliar os resultados das avaliações de integridade dos componentes mais críticos das caldeiras, o Cepel adquiriu um equipamento apropriado para realizar medições de espessura de paredes de tubos, assim como medições de espessura de camada interna de óxidos, mais precisamente a Magnetita. Segundo a literatura [6], a espessura mínima obtida com um sistema desse tipo é de $130 \mu \mathrm{m}$. Os componentes que constituem o sistema de medição são:

- Detector Ultrassônico de Falhas;

- Aplicativo Interno "Gate de Interface";

- Transdutor Ultrassônico de Onda Transversal, frequência de 20MHz;

- Cabo Unifilar;

- Acoplante Ultraviscoso.

Um dos objetivos do presente artigo consiste em validar as medições de espessura de camada de óxido para que este tipo de ensaio possa ser usado com segurança em aplicações de campo. Sendo assim, foram realizados ensaios para avaliar os seguintes itens:

- $\quad$ A precisão do equipamento;

- O efeito do acabamento superficial na qualidade das medidas;

- Correlação entre os ecos obtidos e a camada de óxido presente.

Para validar os resultados obtidos pelo equipamento foram utilizados tubos retirados de superaquecedores e reaquecedores de caldeiras de usinas termelétricas, pois estes são os componentes mais sujeitos apresentar falhas por sobreaquecimento, devido à formação da camada interna de Magnetita. As amostras utilizadas encontravam-se no depósito do laboratório de metalografia do Cepel. A Tabela 1 apresenta os dados dimensionais médios dos tubos analisados.

Tabela 1: Dados dimensionais das amostras de tubos de superaquecedores e reaquecedores de caldeiras usados para medições de camada interna de óxido.

\begin{tabular}{c|c|c}
\hline Amostra & Espessura da parede do tubo (mm) & Diâmetro (mm) \\
\hline A & $10,12 \pm 0,25$ & $44,14 \pm 0,22$ \\
\hline B & $3,51 \pm 0,17$ & $44,21 \pm 0,05$ \\
\hline C & $4,65 \pm 0,37$ & $32,02 \pm 0,19$ \\
\hline
\end{tabular}


Os resultados medidos por ultrassom foram comparados com os resultados obtidos via microscopia ótica. Os testes foram realizados com o intuito de avaliar a faixa confiável de medições do aparelho.

A ideia principal dos ensaios é verificar se o equipamento ultrassônico é capaz de realizar medidas similares as do microscópio ótico através de uma análise estatística dos resultados. A análise estatística mais apropriada para os ensaios realizados, que consistiu em provar a igualdade estatística das medidas de camada de óxido, foi baseada na utilização de testes de hipótese. A ferramenta utilizada para a realização dos testes foi o programa Microsoft Excel ${ }^{\circledR}$.

Para analisar a capacidade de acoplamento do transdutor em função da condição superficial dos tubos, os mesmos foram divididos em quadrantes, conforme a Figura 1 abaixo, na qual os quadrantes A e B possuíam a superfície lixada com lixa de preparação metalográfica de 120mesh, enquanto os quadrantes C e D possuíam a superfície esmerilhada. Foi usado um disco de desbaste do fabricante Bosch, modelo A24RBF na preparação das superfícies esmerilhadas, o objetivo foi apenas remover a camada de óxido externa. Foram realizadas dez medições de espessura de camada de óxido por ultrassom e por microscopia ótica em cada um dos quadrantes das amostras selecionadas.

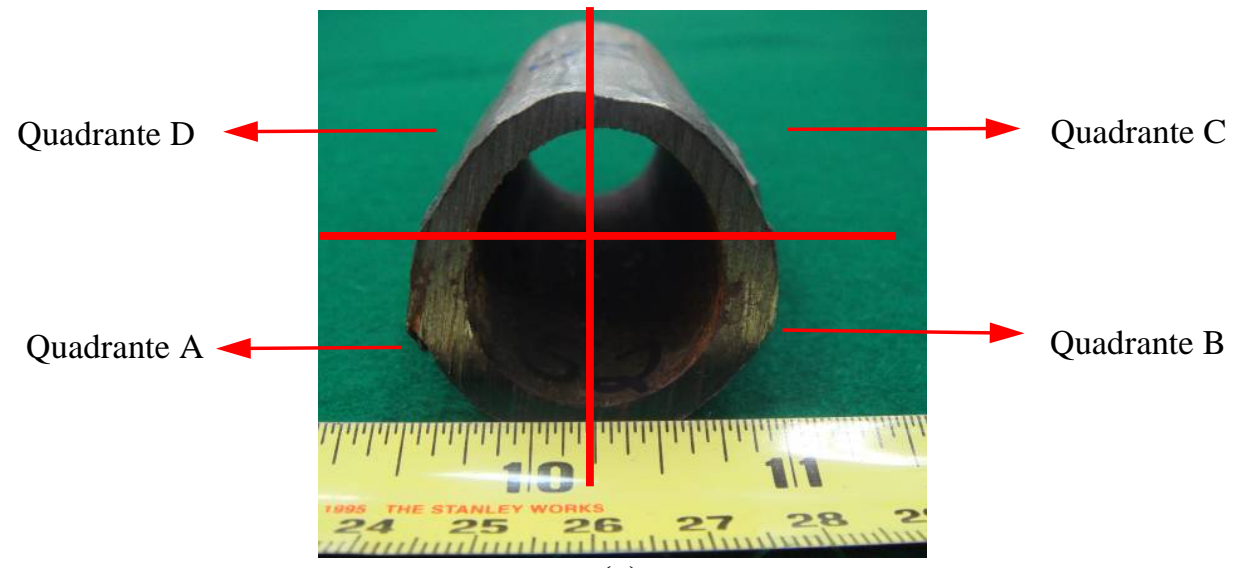

(a)

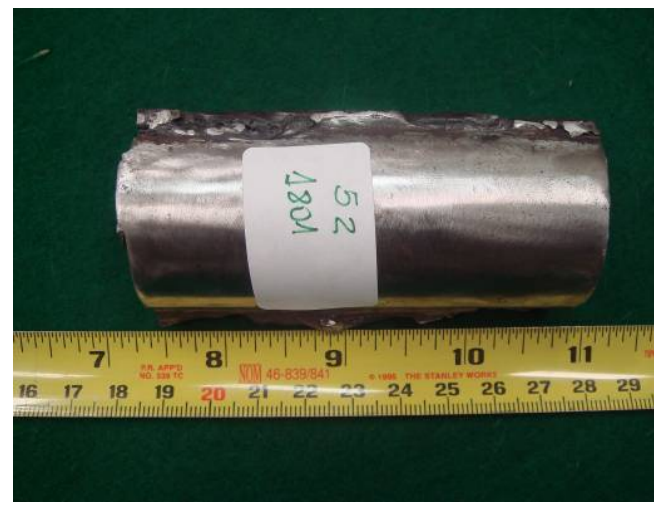

(b)

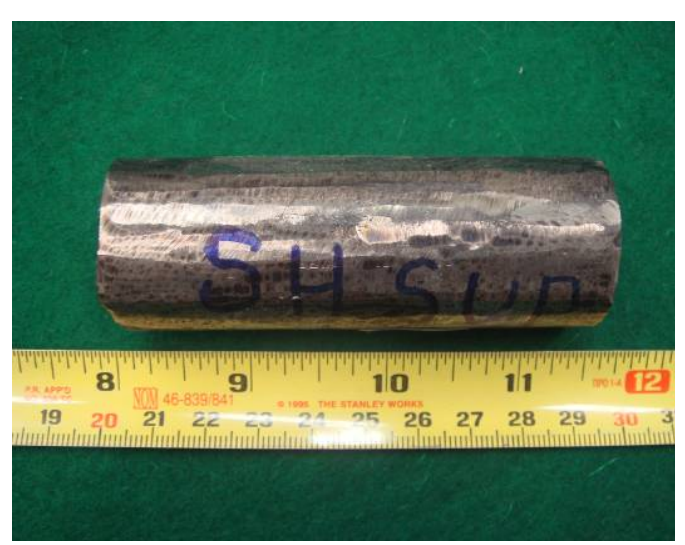

(c)

Figura 1: Tipos de superfícies utilizadas para avaliar o acoplamento do transdutor ultrassônico: (a) vista geral dos quadrantes adotados; (b) detalhes dos quadrantes A e B; (c) detalhe dos quadrantes C e D.

\subsection{Correlação Entre os Sinais Ultrassônicos e a Presença da Camada de Óxido}

Uma análise foi realizada com o intuito de correlacionar a natureza do sinal ultrassônico com a espessura da camada interna de Magnetita presente. Para isso, foram obtidos os ecos provenientes de uma amostra de tubo de caldeira intacto, ou seja, sem a presença da camada de óxido, e das amostras que continham camada interna de óxido. O objetivo foi tentar definir padrões de sinais para realização de análises mais rápidas durante as inspeções de campo. 


\subsection{Estudo de Caso}

O estudo de caso do presente artigo foi realizado com o intuito de estimar a vida remanescente, para o mecanismo de dano por fluência, de dois tubos retirados da serpentina do superaquecedor final de uma caldeira de uma unidade de geração de energia termelétrica no Brasil. O material dos tubos é o aço ASTM A 387 Grau 22, um aço do tipo 2,25Cr-1Mo, muito utilizado em aplicações a alta temperatura. Ambos os tubos operaram por aproximadamente 191.000,00 horas, sendo que um tubo apresentou camada interna inferior a $130 \mu \mathrm{m}$ e o outro uma camada interna superior a $700 \mu \mathrm{m}$. A Figura 2 apresenta um desenho esquemático do componente analisado. Os tubos foram retirados da região denominada $7 \mathrm{X}$, identificada na Figura 2 por uma seta. As condições nominais de pressão e temperatura de operação são $10,8 \mathrm{MPa}\left(110 \mathrm{Kgf} / \mathrm{cm}^{2}\right)$ e $510^{\circ} \mathrm{C}$, respectivamente. O diâmetro externo e a espessura nominal dos tubos são 31,8mm e 5,6mm, respectivamente.

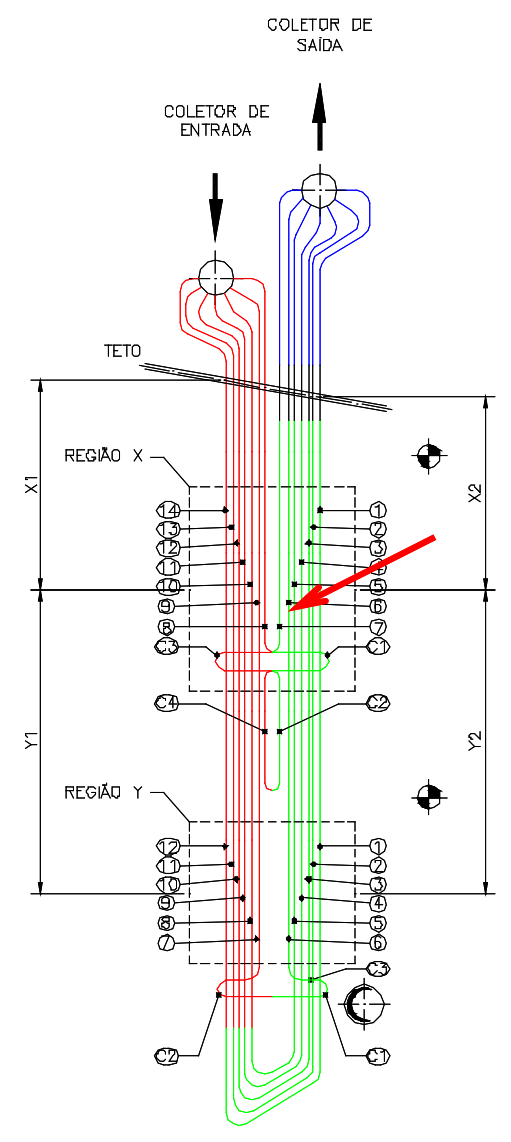

Figura 2: Desenho esquemático do superaquecedor utilizado no estudo de caso.

A Figura 3 apresenta as amostras recebidas para realização das medidas em laboratório por microscopia ótica e pelo método ultrassônico.

No caso específico da camada interna de óxido de tubos de superaquecedores e reaquecedores de caldeiras, já existe um método consagrado na literatura [3]. O método consiste em uma abordagem empírica para avaliação de vida remanescente de tubos sujeitos ao mecanismo de degradação por fluência. Sua grande vantagem é utilizar dados rapidamente disponibilizados pelos sistemas de monitoramento e supervisão das unidades geradoras. O método baseia-se na observação experimental da dependência do crescimento da camada de óxido que se deposita na parede interna dos tubos (local por onde escoa o vapor) com a temperatura de metal do tubo e o tempo de exposição a esta temperatura. Conhecendo-se o número total de horas até o momento presente e medindo-se a espessura da camada de óxido em amostras de tubos durante paradas programadas da unidade, obtêm-se uma estimativa da temperatura média efetiva de metal a qual foram submetidos os tubos.

Medidas de dados geométricos dos tubos tais como o diâmetro externo e/ou a espessura da parede durante as paradas programadas, permitem estimar a taxa de desgaste da parede externa e, consequentemente, a redução do raio externo dos tubos ao longo do tempo. Conhecendo a geometria dos tubos e a pressão 
interna do vapor obtém-se então a evolução das tensões mecânicas na parede dos tubos ao longo do tempo. Comparando a curva real de tensão a qual estão sujeitos os tubos à curva de resistência à fluência do material que os constitui, é possível calcular a vida remanescente à fluência.

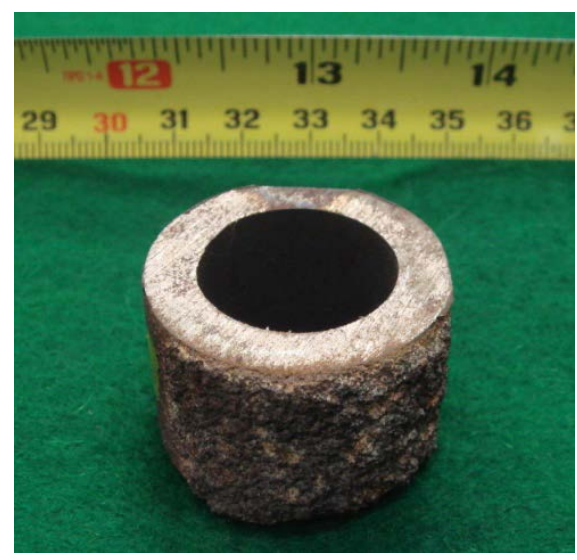

(a)

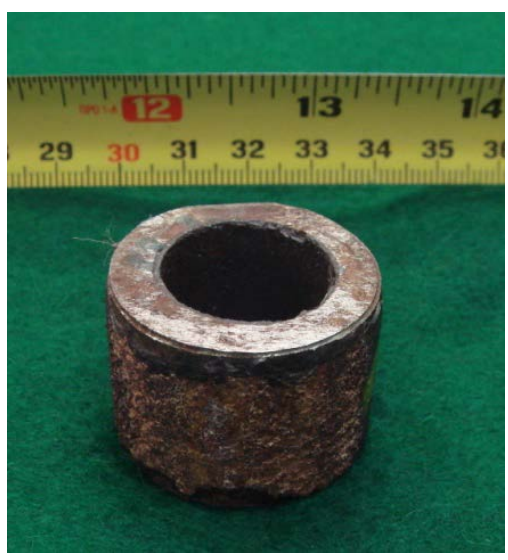

(b)

Figura 3: Amostras dos tubos da serpentina do superaquecedor da caldeira de geração de energia

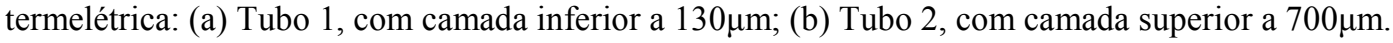

O método de cálculo para estimativa de vida residual, baseado em medições de camada interna de Magnetita, pode ser divido em três módulos [3]. Os módulos e os dados de entrada necessários para cada um deles são apresentados a seguir: módulo são:

Módulo 1 - Dados geométricos do tubo sob investigação e carregamento. Os dados de entrada neste

- Os raios interno e externo do tubo no instante de tempo $t=0$ (isto é, os valores nominais de projeto ou informados antes do início da operação do equipamento);

- O número de horas de operação do equipamento até a parada;

- O valor mínimo medido do raio externo do tubo após a parada da unidade;

- A pressão interna do vapor.

Os dados de saída são a taxa de desgaste na parede externa do tubo, e a variação do raio externo do tubo em função do tempo.

Módulo 2 - Estimativa da evolução da espessura da camada de óxido na parede interna do tubo e da temperatura efetiva de metal. Os dados de entrada neste módulo são:

- O valor médio da espessura da camada de óxido medida em campo após a parada da unidade;

- Modelo adotado para o crescimento da camada de óxido: linear ou quadrático.

O modelo quadrático foi o adotado, pois esse apresentou resultados mais conservadores.

O dado de saída é a temperatura média efetiva de metal no instante de tempo que corresponde ao tempo de operação até o momento da análise.

Módulo 3 - Estimativa do dano total acumulado e da vida remanescente. Neste módulo, pode-se obter a vida remanescente por duas maneiras. Na primeira, são calculadas as tensões mecânicas representativas e os pontos de interseção entre a curva de tensão real a qual está sujeito o tubo sob investigação e as curvas de resistência à fluência do aço analisado. Na segunda, a vida remanescente é calculada a partir do dano total acumulado até o momento da parada para inspeção. Utilizando a regra de acúmulo linear de Robinson pode-se calcular o dano total por fluência acumulado até o instante de tempo correspondente ao tempo de operação. Para ambas, foram utilizadas as curvas de resistência à fluência do material ASTM A 387 Grau 22. A literatura recomenda a utilização do resultado mais conservador. 


\section{RESULTADOS E DISCUSSÃO}

\subsection{Validação das Medições Realizadas com Equipamento Ultrassônico}

A Figura 4 apresenta os valores de espessura de camada interna de óxido obtida pelo equipamento ultrassônico (US) e por microscopia ótica (MO) ao longo dos quadrantes das amostras A, B e C.

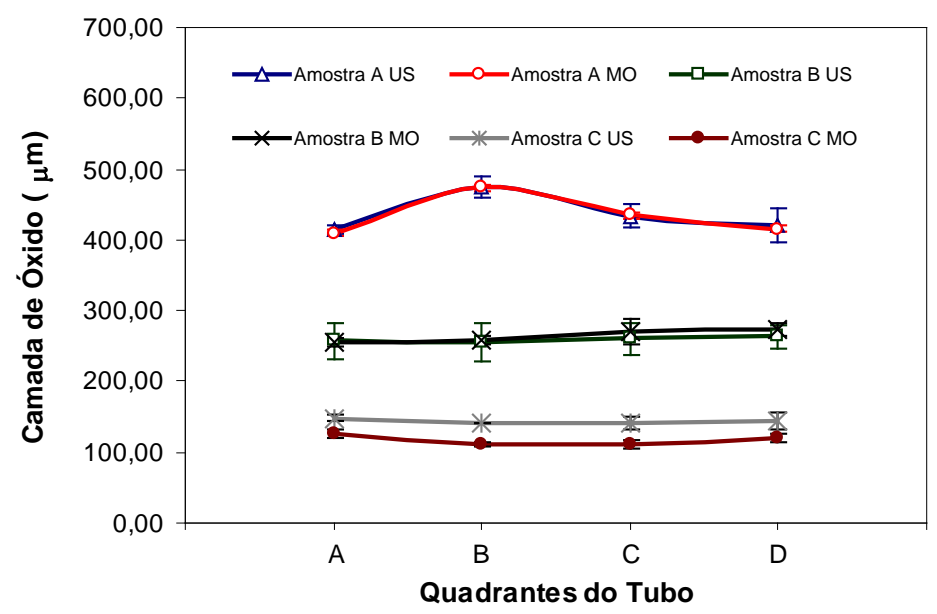

Figura 4: Valores de camada de óxido obtidos pelo equipamento ultrassônico e por microscopia ótica ao longo dos quadrantes das amostras A, B e C.

É possível notar que as medidas realizadas por ultrassom e por microscopia ótica nas amostras A e B apresentaram valores muito próximos e comportamentos similares ao longo dos quadrantes. No caso da amostra C, pode-se observar que os valores medidos por microscopia ótica apresentam uma leve variação e são menores que os valores obtidos por ultrassom.

Para realização dos testes de hipótese, as medidas obtidas nos quadrantes A e B pelo equipamento ultrassônico e por microscopia ótica foram agrupadas e comparadas entre si. O mesmo procedimento foi realizado para os quadrantes $\mathrm{C}$ e $\mathrm{D}$. A Tabela 2 apresenta o resultado do teste de hipótese realizado na região $\mathrm{AB}$ da amostra $\mathrm{A}$.

Tabela 2: Teste de hipótese para igualdade das médias das medidas de camada interna de óxido realizado com o equipamento ultrassônico e com o microscópio ótico na amostra A, região AB.

\begin{tabular}{c|c|c}
\hline \multicolumn{3}{c}{ Amostra A - Região AB } \\
\hline Média & Ultrassom & Microscópio Ótico \\
\hline Variância & 438 & 441,64393 \\
\hline Observações & 1084,444444 & 1145,905681 \\
\hline Hipótese da diferença de média & 10 & 10 \\
\hline Gl & 0 & - \\
\hline Stat t & 18 & - \\
\hline $\mathbf{P}(\mathbf{T}<=$ t) uni-caudal & $\mathbf{- 0 , 2 4 3 9 9 6 4 4 7}$ & - \\
\hline $\mathbf{t}$ crítico uni-caudal & 0,404996495 & - \\
\hline $\mathbf{P}(\mathbf{T}<=\mathbf{t})$ bi-caudal & 1,734063592 & - \\
\hline $\mathbf{t}$ crítico bi-caudal & 0,80999299 & - \\
\hline
\end{tabular}

É possível notar no resultado apresentado na Tabela 2 que o valor calculado do teste estatístico (Stat t) é menor que os valores críticos, tanto para teste uni-caudal quanto para o bi-caudal, sendo este o escolhido para a análise realizada com as medidas de camada de óxido. Sendo assim, não foi possível rejeitar as hipóteses das medidas serem estatisticamente diferentes. Pode-se aceitar com $95 \%$ de certeza que as medidas 
de camada de óxido realizadas pelo equipamento ultrassônico e pelo microscópio ótico são estatisticamente iguais.

Os mesmos resultados foram obtidos para os outros quadrantes da amostra A, assim como para todos os quadrantes da amostra B.

No caso da amostra C, os valores calculados foram superiores aos valores críticos obtidos nos testes. Sendo assim, a hipótese das medidas serem estatisticamente iguais deve ser rejeitada. Não se pode afirmar que as medições são estatisticamente iguais para o caso dessa amostra em nenhuma das regiões analisadas. Esse resultado era esperado. De acordo com literatura [6], sistemas para medição de camada de óxido constituídos de aparelhos manuais para deteção de falhas e transdutores de ondas cisalhantes com frequência de $20 \mathrm{MHz}$, apresentam um limite mínimo de medição de 125 a $140 \mu \mathrm{m}$, dependendo das condições de ajuste do equipamento. No caso da amostra em questão, as médias das espessuras de camada de óxido medidas por

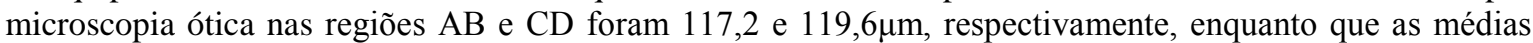
apresentadas pelo equipamento ultrassônico foram de 144 e $143 \mu \mathrm{m}$, mostrando que o limite mínimo do equipamento foi atingido.

Para realizar medições abaixo dessa espessura seriam necessários transdutores de frequências mais elevadas, para possibilitar a distinção entre os dois ecos ultrassônicos necessários nas medições. Esses tipos de transdutores necessitam de condições mais controladas de ensaio assim como uma preparação superficial de elevada qualidade para que seja garantido um bom acoplamento, o que torna suas aplicações em campo mais limitadas [6].

Com relação ao efeito do acabamento superficial nos tubos para realização das medidas, foi possível notar um melhor acoplamento nas regiões que foram esmerilhadas e posteriormente lixadas em todas as amostras. No entanto, não houve maiores dificuldades de acoplamento na região que foi apenas esmerilhada, mesmo nas medições realizadas para avaliar o limite mínimo de medição do equipamento. Dessa maneira, pode-se ganhar tempo em inspeções de campo, desde que a superfície esmerilhada esteja similar a das amostras analisadas em laboratório.

\subsection{Correlação Entre os Sinais Ultrassônicos e a Presença da Camada de Óxido}

A Figura 5 apresenta os sinais ultrassônicos, obtidos no modo de observação A scan do equipamento para uma amostra de tubo intacta e as amostras com camada interna de óxido. Os ecos das amostras A, B e C foram apresentados em ordem crescente em relação à espessura da camada.

A partir dos resultados apresentados é possível notar que para espessuras inferiores a $130 \mu \mathrm{m}$, não é possível identificar uma separação entre os ecos referentes às interfaces aço/camada de óxido e camada de óxido/ar. Sendo assim, é preciso levar em consideração que os tubos que apresentarem tal espessura no campo podem possuir uma espessura inferior à medida, porém não detectada pelo equipamento. Para espessuras maiores, os ecos tendem a apresentar um espaçamento maior, facilitando a medição da camada.

\subsection{Estudo de Caso}

A Figura 6 apresenta os valores de espessura de camada interna de óxido medida por microscopia ótica e por ultrassom nas amostras recebidas. Para o caso do Tubo 1, foi obtido um valor médio de $130 \mu \mathrm{m}$ por ultrassom, indicando o limite inferior do equipamento, enquanto que a média obtida por microscopia ótica foi de $34 \mu \mathrm{m}$. Para o caso do Tubo 2, os valores obtidos pelas duas técnicas foram considerados estatisticamente iguais, com 95\% de certeza, através de testes de hipóteses realizados com as médias de cada um dos quadrantes.

O quadrante denominado $\mathrm{C}$ do Tubo 2 apresentou uma camada com espessura média de $993 \mu \mathrm{m}$. Essa região do tubo é a que fica exposta à região dos queimadores da caldeira, ou seja, exposta a maior carga térmica. A temperatura mais elevada nessa região do tubo pode ter contribuído para um crescimento mais pronunciado da camada interna de Magnetita.

É importante lembrar que a camada se forma a partir da parede do tubo, portanto, além de atuar como um isolante térmico que aumenta a temperatura do metal, a camada também atua reduzindo a espessura do tubo e, por conseguinte, a seção resistente do mesmo.

Para realização da estimativa de vida remanescente, foram utilizados os valores de camada interna de Magnetita de $130 \mu \mathrm{m}$ para o Tubo 1, pois este foi o valor mínimo medido pelo equipamento. Mesmo o tubo apresentando uma camada inferior à adotada, o valor foi utilizado para simular uma análise realizada em campo, no qual a retirada de uma amostra para medição por microscopia ótica não seria viável. O valor $933 \mu \mathrm{m}$, referente à média do quadrante $\mathrm{C}$, exposto ao lado da chama da caldeira, foi utilizado para o Tubo 2 . A Tabela 3 apresenta de forma resumida os principais resultados obtidos pela metodologia de cálculo.

Como citado anteriormente, a camada se forma à custa da espessura da parede do tubo, portanto, já era esperada uma maior tensão mecânica para o tubo contendo a camada mais elevada. 
A temperatura calculada para o metal do Tubo 2, devido à presença de uma elevada camada de óxido, que atua como um isolante térmico durante a operação da caldeira, foi de $601,8{ }^{\circ} \mathrm{C}$. Para o Tubo 1 a temperatura manteve-se de acordo com a de operação nominal, mostrando que uma camada de $130 \mu \mathrm{m}$ não influi na troca térmica do tubo.

Tabela 3: Principais resultados obtidos pela metodologia de cálculo para estimativa de vida remanescente por fluência baseada na medição da camada interna de óxido.

\begin{tabular}{c|c|c|c|c|c}
\hline Tubo & $\begin{array}{c}\text { Camada de } \\
\text { Óxido }(\boldsymbol{\mu m})\end{array}$ & $\begin{array}{c}\text { Tensão Representativa } \\
\mathbf{( M P a )}\end{array}$ & $\begin{array}{c}\text { Temperatura } \\
\text { Efetiva }\left({ }^{\circ} \mathbf{C}\right)\end{array}$ & $\begin{array}{c}\text { Vida } \\
\text { Remanescente } \\
\text { Método 1 (h) }\end{array}$ & $\begin{array}{c}\text { Vida } \\
\text { Remanescente } \\
\text { Método 2 (h) }\end{array}$ \\
\hline $\mathbf{1}$ & 130 & 28,2 & 509,8 & $2,04 \times 10^{6}$ & $1,05 \times 10^{6}$ \\
\hline $\mathbf{2}$ & 993 & 31,6 & 601,8 & $2,57 \times 10^{5}$ & $1,06 \times 10^{5}$ \\
\hline
\end{tabular}

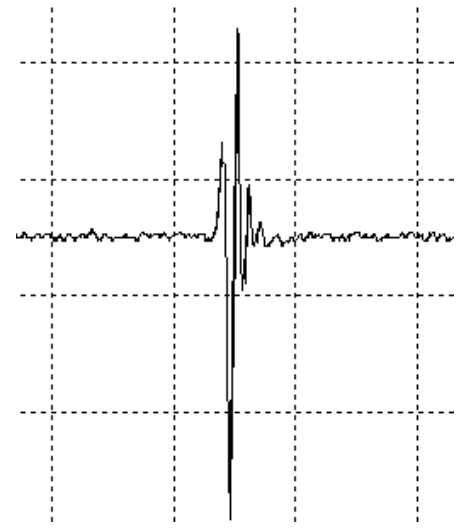

(a)

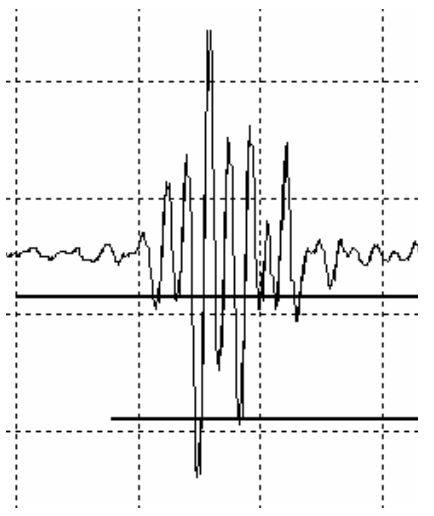

(c)

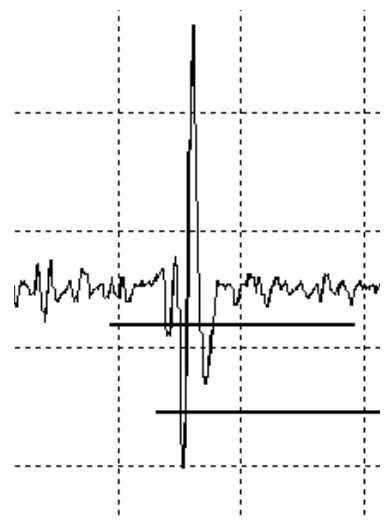

(b)

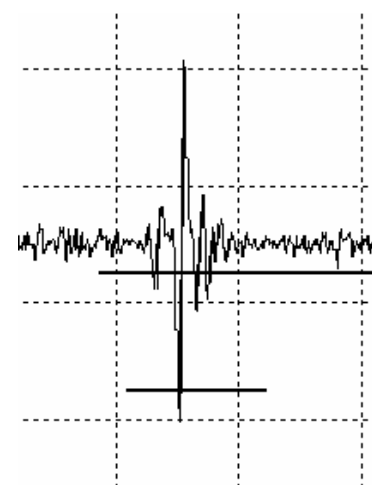

(d)

Figura 5: Sinais ultrassônicos obtidos das amostras: (a) Tubo Intacto; (b) Amostra C com camada inferior a $130 \mu \mathrm{m}$; (c) Amostra B com camada média de 260 $\mu$ m; (d) Amostra A com camada média de $430 \mu \mathrm{m}$. 


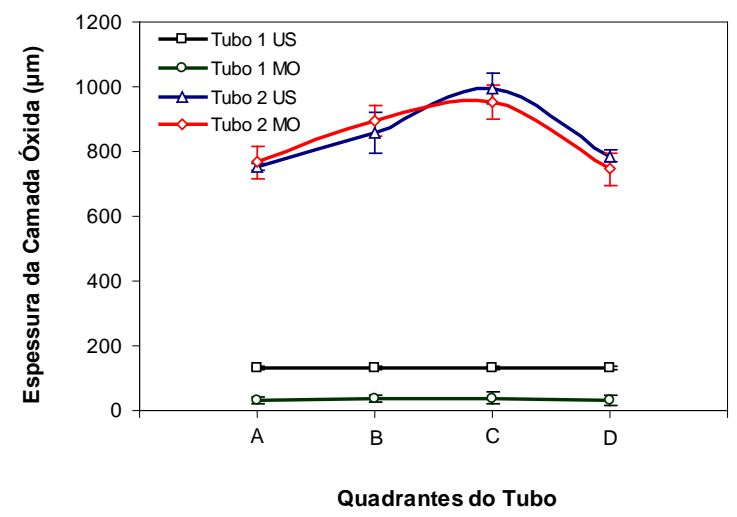

Figura 6: Valores de camada de óxido obtidos pelo equipamento ultrassônico e por microscopia ótica ao longo dos quadrantes dos Tubos 1 e 2 recebidos.

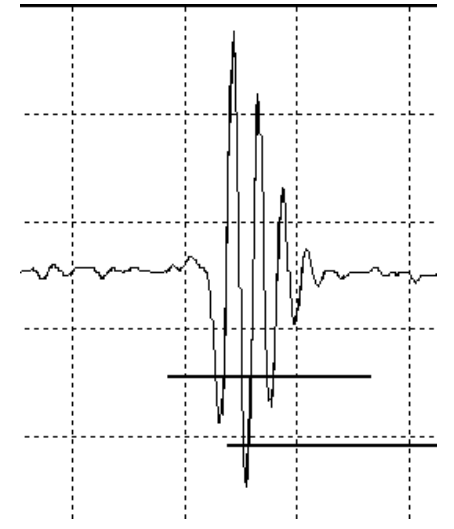

(a)

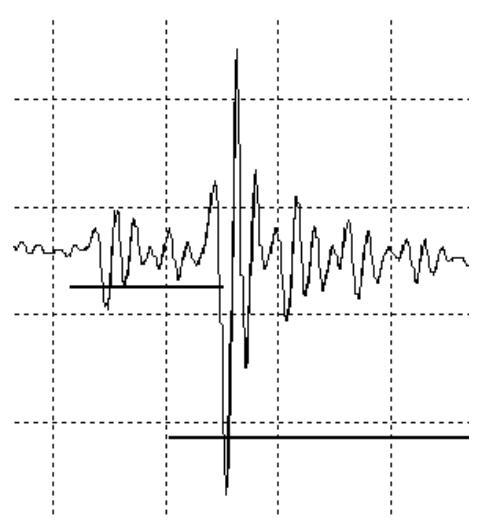

(b)

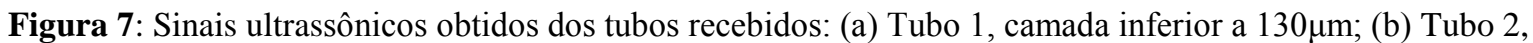
com camada superior a $700 \mu \mathrm{m}$.

A Figura 7 apresenta os sinais ultrassônicos obtidos nos Tubos 1 e 2. Nota-se uma semelhança entre as Figuras 5(b) e 7(a), na qual o equipamento mede uma camada com valor de $130 \mu \mathrm{m}$. Na verdade, esse valor é obtido quando as portas de medição do equipamento são colocadas na primeira e segunda inflexões do sinal, respectivamente. Esse tipo de colocação das portas não significa que o equipamento está detectando os dois ecos necessários para medição da camada interna de óxido, como os da Figura 7(b).

Os resultados acima mostram claramente como a camada interna de óxido pode influenciar a vida remanescente do material. É possível notar que a presença de uma camada espessa foi capaz de reduzir em aproximadamente uma ordem de grandeza a vida remanescente do Tubo 2, quando o mesmo é comparado com o Tubo1, para ambos os métodos de cálculo. Seguindo-se a recomendação da literatura de adotar o

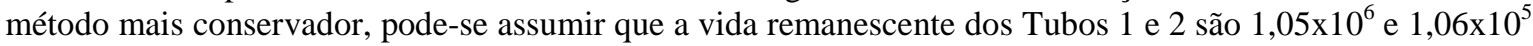
horas, respectivamente.

Uma análise foi realizada para verificar qual espessura de camada de Magnetita seria necessária para causar um dano severo aos tubos no componente analisado. Considerando-se as condições geométricas e de operação da caldeira no momento do estudo, foi constatado que uma camada de $1500 \mu \mathrm{m}$ e uma de $1350 \mu \mathrm{m}$ seriam necessárias para extinguir a vida dos Tubos 1 e 2, respectivamente, sob o regime de fluência.

A literatura enfatiza que nem sempre as medições de camada de óxido podem fornecer um histórico correto de temperatura do metal ao longo da sua operação. Isto se deve ao fato de que muitas vezes a camada sofre quebras devido a diversos motivos, como vibrações, choques entre tubos das serpentinas ou limpezas químicas [7]. Segundo JAUHIAINEIN et al. [8] é mais provável encontrar camadas quebradas em plantas mais antigas. Apesar de causarem problemas, as quebras das camadas podem aumentar a vida residual dos tubos, visto que o efeito isolante causado pela presença da camada é reduzido quando esta diminui sua espessura. 
Em vista das informações obtidas, é recomendável uma monitoração, através de medições de espessura de camada de óxido de forma continuada, para uma análise mais precisa do dano por fluência em tubos de superaquecedores e reaquecedores de caldeiras utilizadas em plantas industriais.

\section{CONCLUSÕES}

Com base nos resultados obtidos, pode-se concluir:

- As medições realizadas pelo equipamento ultrassônico puderam ser consideradas estatisticamente iguais às realizadas por microscopia ótica para espessuras de camada de óxido acima de $130 \mu \mathrm{m}$, limite mínimo de medição do equipamento na configuração atual;

- Com relação ao efeito do acabamento superficial nos tubos para realização das medidas, foi possível notar um melhor acoplamento nas regiões que foram esmerilhadas e posteriormente lixadas em todas as amostras. No entanto, não houve maiores dificuldades de acoplamento na região que foi apenas esmerilhada, mesmo nas medições realizadas para avaliar o limite mínimo de medição do equipamento;

- Existe uma correlação entre a presença da camada de óxido e o formato do sinal ultrassônico, sendo que para espessuras de camada próximas ao limite inferior torna-se mais difícil distinguir os ecos das interfaces aço/óxido e óxido/ar. Para espessuras acima de $140 \mu \mathrm{m}$ em diante é possível identificar os ecos de cada uma das interfaces com maior facilidade;

- A partir dos resultados obtidos para estimativa de vida residual, pelo mecanismo de dano fluência, foi possível constatar que uma camada de $130 \mu \mathrm{m}$ não contribuiu de maneira deletéria para o Tubo1. Para o Tubo 2, com uma camada acima de $700 \mu \mathrm{m}$, foi estimada uma vida remanescente com uma ordem de grandeza a menos que a do Tubo 1, indicando que uma camada espessa de Magnetita pode influir significativamente na vida residual de tubos de superaquecedores e reaquecedores de caldeiras;

- A utilização da metodologia descrita no presente artigo pode ser aplicada no campo sem maiores dificuldades, auxiliando de maneira efetiva a tomada de decisões durante uma parada programada para inspeção de caldeiras de plantas industriais.

\section{BIBLIOGRAFIA}

[1] ASM, METALS HANDBOOK, Failure analysis and prevention - vol. 11, Ohio, Metals Park, ASM International, 2002.

[2] PATERSON, S.R., MOSER, R., RETTING, T.W., "The oxidation of boiler tubes”, In: Proceedings of an International Conference on Interaction of Iron-Based Materials with Water and Steam, pp. 8.18.25, Heidelberg, Jun. 1992.

[3] PATERSON, S.R., RETTING, T.W., Remaining life assessment of superheater and reheater tubes, In: EPRI CS-5564 Report, Electric Power Research Institute (EPRI), Palo Alto, Califórnia, 1988.

[4] HAWKINS, B.A., AUBREY, R.P., JAMISON, T.D., "Use measurements of oxide scale thickness to maintain high availability”, In: Proceedings of the International Exhibition \& Conference for the Power Generation Industries, pp. 575-589, New Orleans, Dez. 1989.

[5] WARDLE, T.J., "Creep-rupture assessment of superheater tubes using nondestructive oxide thickness measurements", In: Proceedings of the International Conference on Life Management and Life Extension of Power Plant, China, 2000.

[6] LABRECK, S., KASS, D., NELLIGAN, T., "Ultrasonic thickness measurement of internal oxide scale in steam boilers”, In: Proceedings of the European Conference on Non-Destructive Testing (ECNDT), Mo.2.8.3, Berlin, Sept. 2006.

[7] VISWANATHAN, R., Damage mechanisms and life assessment of high-temperature components, 1 ed., Ohio, Metals Park, ASM International, 1989.

[8] JAUHIAINEN, P., YLI-OLLI, S., NYHOLM, A., et al., "Impact of oxidation on creep life of superheaters and reheaters", In: Proceedings of the International ECCC Creep Conference, pp. 320328, Zurique, Apr. 2009. 